\title{
PREVALENCE OF THE RHEUMATOID FACTOR AND ITS INFLUENCE ON THE CLINICAL PROFILE AND
} GRAVITY OF SYSTEMIC SCLEROSIS

Isadora Dal Pont (Hospital Universitário Evangélico Mackenzie, Curitiba, PR, Brasil), Jady Elen de Pontes (Rheumatology Unit - Hospital Universitário Evangélico Mackenzie, Curitiba, PR, Brasil), Thiago Alberto

Fernandes Gomes dos Santos (Rheumatology Unit - Hospital Universitário Evangélico Mackenzie, Curitiba, PR, Brasil), Patricia Martin (Rheumatology Unit - Hospital Universitário Evangélico Mackenzie ; Escola de Medicina - Pontificia Universidade Católica do Paraná (PUCPR), Curitiba, PR, Brasil), Pedro Ming Azevedo (Rheumatology Unit - Hospital Universitário Evangélico Mackenzie, Curitiba, PR, Brasil), Thelma Laroca Skare (Rheumatology Unit - Hospital Universitário Evangélico Mackenzie, Curitiba, PR, Brasil)

\section{BACKGROUND}

Systemic sclerosis (SSc) is a diffuse autoimmune connective tissue disease, in which cutaneous fibrosis associated with various forms of visceral involvement is identified. Serum autoantibodies are found in $80 \%$ or more of the patients; the classical are anti-centromere (ACA) and anti-topoisomerase I (anti-Scl70). Several other autoantibodies may be present in ES, although at a lower frequency and without connotation of diagnostic markers. Rheumatoid factor (RF), found in 30 to $40 \%$ of patients.

\section{MATERIALS AND METHODS}

Retrospective study of 108 charts of patients with SSc from the last 20 years of the HUEMC Rheumatology Outpatient Clinic to analyze the serum IgM-FR values. Epidemiological data (age at onset of ES, race, sex, smoking, and alcohol consumption) were also collected; clinical findings (clinical form of disease, Medsger's Index, modified Rodnan's Index and findings such as Raynaud's Phenomenon, stellar scars, telangiectasia, microstomia, altered esophageal motility, pulmonary fibrosis and hypertension, myosites, myocarditis, renal scleroderma, arthralgia, arthritis and tendinopathies) and serological data from other autoantibodies (FAN, ACA, anti-Scl-70, anti-Ro, anti-RNP and anti-La). Patients with positive RF were compared to the negative RF.

\section{RESULTS}

The prevalence of RF in the sample studied was $36.11 \%$. The RF did not change the clinical and epidemiological profile including joint symptoms (all $p=n s$ ). Regarding the serological profile, there was a statistically significant difference in anti-Scl-70 $(p=0.02)$. Negative FR patients have eight times (O.R. = 8.3) more chances of having this autoantibody than the FR positive patients.

\section{CONCLUSION}

The prevalence of RF in ES patients was $36.11 \%$, higher than the prevalence found in most other studies, but within the expected range for this group of patients. There was no statistically significant difference when comparing the FR positive and negative RR groups for the epidemiological and clinical profiles. Regarding the autoantibody profile, a significant statistical difference was found between the groups regarding anti-Scl-70. 